\title{
Low-Energy Dislocation Structure (LEDS) character of dislocation boundaries aligned with slip planes in rolled aluminium
}

Winther, Grethe; Hong, Chuanshi; Huang, Xiaoxu

Published in:

Philosophical Magazine

Link to article, DOI:

10.1080/14786435.2015.1033488

Publication date:

2015

Document Version

Peer reviewed version

Link back to DTU Orbit

Citation (APA):

Winther, G., Hong, C., \& Huang, X. (2015). Low-Energy Dislocation Structure (LEDS) character of dislocation boundaries aligned with slip planes in rolled aluminium. Philosophical Magazine, 95(13), 1471-1489.

https://doi.org/10.1080/14786435.2015.1033488

\section{General rights}

Copyright and moral rights for the publications made accessible in the public portal are retained by the authors and/or other copyright owners and it is a condition of accessing publications that users recognise and abide by the legal requirements associated with these rights.

- Users may download and print one copy of any publication from the public portal for the purpose of private study or research.

- You may not further distribute the material or use it for any profit-making activity or commercial gain

- You may freely distribute the URL identifying the publication in the public portal 
The Version of Record of this manuscript has been published on 29 Apr. 2015 and is available in Philosophical Magazine 95(13) 1471-1489. http://www.tandfonline.com/ DOI: 10.1080/14786435.2015.1023488

\title{
Low-Energy Dislocation Structure (LEDS) character of dislocation boundaries aligned with slip planes in rolled aluminium
}

G. Winther ${ }^{\mathrm{a} *}$, C.S. Hong ${ }^{\mathrm{b}}$, X. Huang ${ }^{\mathrm{b}}$

${ }^{a}$ Department of Mechanical Engineering, Technical University of Denmark, DK-2800 Kgs. Lyngby, Denmark

${ }^{b}$ Danish-Chinese Center for Nanometals, Section for Materials Science and Advanced Characterization, Department of Wind Energy, Technical University of Denmark, DK-4000 Roskilde, Denmark

*Corresponding author. Email: grwi@mek.dtu.dk

\begin{abstract}
For the specific slip geometry of two sets of coplanar systems (a total of four systems) in fcc metals, the range of dislocation networks in boundaries aligned with one of the two active slip planes is predicted from the Frank equation for boundaries free of long-range elastic stresses. Detailed comparison with experimental data for eight dislocation boundaries in cold-rolled aluminium grains of the $45^{\circ}$ ND rotated Cube orientation is conducted. It is concluded that the boundaries are Low Energy Dislocation Structures (LEDS), which are in good agreement with the Frank equation while also lowering the energy by dislocation reactions. Cross slip plays a role in the boundary formation process.
\end{abstract}

\section{Keywords}

Dislocation boundaries; Dislocations; Dislocation theory; Low energy dislocation structures; Slip systems; Fcc crystal structure

\section{Introduction}

Deformation-induced dislocation boundaries in metals of intermediate to high stacking fault energy have been studied intensely over the past decades, partly due to their contribution to mechanical properties [1-6] and partly because of the scientifically intriguing process of dislocation self-assembly into a fairly regular pattern of low-angle dislocation boundaries [7-11].

Two main categories of dislocation boundaries have been identified based on their morphological appearance: extended planar boundaries and cell boundaries [12]. The planar boundaries are believed to be geometrically necessary boundaries (GNBs) in the sense that they separate domains in the material with different slip system activity. The cell boundaries are assumed to originate from statistically 
trapped dislocations and are also called incidental dislocation boundaries (IDBs). At low and intermediate strains $(0.05<\varepsilon<1)$ the planar GNBs align with specific crystallographic planes $[13,14]$. GNBs aligned with slip planes are referred to as Type 1 while GNBs aligning with other crystallographic planes are of Type 3 . Type 2 has no GNBs but only cell boundaries.

The type of the dislocation structure evolving within a grain depends on the crystallographic grain orientation as demonstrated for both fcc [14-18] and bcc [19-21] metals. This grain orientation dependence has been explained by a dependence on the active slip systems, and prediction of the dislocation structure type, and especially the detailed alignment of the GNBs in Types 1 and 3 is now possible in fcc metals based on the slip systems [22-24].

Slip-plane-aligned boundaries have been the subject of extensive studies, primarily in copper single crystals oriented for single slip in the early stages of tensile deformation [25-27]. By contrast, the present paper focuses on well-developed boundaries in grains with coplanar slip systems in rolled aluminium polycrystals.

In rolled aluminium, GNBs of Type 1 have consistently been found in grains/crystals with two sets of coplanar slip systems (i.e. a total of four systems) [28,29]. The GNBs align closely with the active slip planes. This combination of slip geometry and GNB alignment is characteristic for grain orientations belonging to the $\alpha$-fibre of the fcc rolling texture, i.e. grain orientations between the Goss ( $45^{\circ}$ RD rotated Cube) and the Brass orientation, as well as in grains in the $45^{\circ} \mathrm{ND}$ rotated Cube orientation. For specific examples, please see [30] and [31] for single crystals and polycrystals, respectively, of Brass orientation, [32] and [15] for the Goss orientation, and [33,34] and [35] for the $45^{\circ} \mathrm{ND}$ rotated Cube orientation.

According to the principle of Low Energy Dislocation Structures (LEDS) the dislocations arrange themselves in the configuration with the lowest energy that is accessible to them [8]. The driving force for the assembly of dislocations in boundaries is believed to be that the dislocations screen each other's elastic stress fields. The characteristics, i.e. Burgers vector and line direction, of the dislocations in a boundary free of long-range elastic stresses are related to the parameters characterising the boundary itself, i.e. the crystallographic boundary plane and the misorientation across the boundary, by the Frank equation [36].

The aim of the present paper is to systematically predict and analyse the range of possible solutions to the Frank equation for slip-plane-aligned boundaries constructed from dislocations selected from two sets of coplanar slip systems in the fcc crystal structure. The theoretical predictions are compared with the experimental observations from a previous paper [31] on the detailed characterisation of dislocation networks in GNBs in grains of the $45^{\circ} \mathrm{ND}$ rotated Cube orientation in $10 \%$ cold-rolled aluminium of high purity.

\section{Methods and terminology}

To facilitate comparison with the experimental data reported in the previous paper [31] for the $45^{\circ} \mathrm{ND}$ rotated Cube orientation, the same slip system and Burgers vector notation as that used in [31] is employed here. This notation is summarised in Figure 1, which also defines the colour code used to 
represent dislocations with the six fcc Burgers vectors of the $1 / 2<101>$ type in subsequent figures. In Figure 1 the two active slip planes are marked in grey and the slip involves glide of dislocations with Burgers vectors b1 and $\mathbf{b 2}$ on (111) and b4 and $\mathbf{b 5}$ on (11 $\overline{1}$ ). These are also the two slip planes with which the GNBs align. Due to the symmetry of the slip systems and GNB planes, only GNBs aligned with (111) are considered throughout the theoretical analysis. Equivalent solutions for the (11 $\overline{1})$ plane obviously exist and examples of these are given in connection with the comparison with experimental data.

(a)

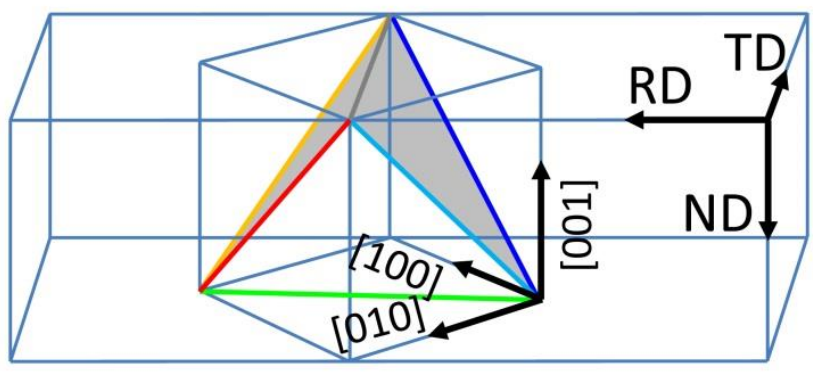

(b)

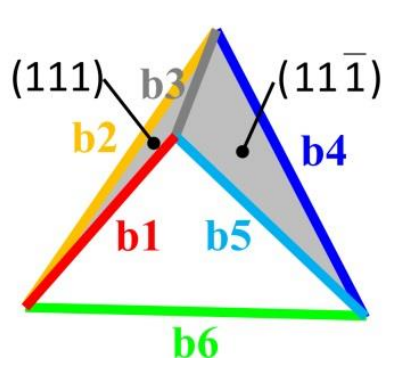

$\mathbf{b} 1=[10 \overline{1}] / 2$

b2 $=[01 \overline{1}] / 2$

b3 $=[1 \overline{1} 0] / 2$

b4 $=[101] / 2$

b5 $=[011] / 2$

b6 $=[110] / 2$

Figure 1. Definition and colour coding of Burgers vectors and the relation to the active slip systems in the rolled $45^{\circ} \mathrm{ND}$ rotated Cube orientation. (a) RD, TD and ND refer to the rolling, transverse and normal directions of rolling geometry, respectively. Slip planes of the coplanar slip systems predicted active are colored gray. (b) Color code for schematic illustration of dislocations based on their Burgers vectors. From [31].

\subsection{LEDS}

The dislocations in a boundary that is free of long-range elastic stresses fulfil the Frank equation [36]:

$$
\sum \rho_{\mathrm{i}} \mathbf{b}_{\mathbf{i}}\left\{\left(\mathbf{n} \times \xi_{\mathbf{i}}\right) \cdot \mathbf{V}\right\}=2 \sin \theta / 2 \mathbf{V} \times \mathbf{a}
$$

where $\mathbf{b}_{\mathbf{i}}$ and $\xi_{\mathbf{i}}$ are the Burgers vector and line direction of dislocation set i. $\rho_{\mathrm{i}}$ is the planar dislocation density defined as length of dislocation line per unit area for each set. The vector $\mathbf{n}$ is the boundary plane normal, and $\mathbf{V}$ is any vector in the boundary plane. The symbols a and $\theta$ are the crystallographic misorientation axis and angle, respectively, across the boundary.

With three sets of dislocations with linearly independent Burgers vectors a solution to Equation (1) exists for any combination of boundary plane $\mathbf{n}$ and misorientation axis $\mathbf{a}$, provided the dislocations can move freely out of their slip plane by climb. Due to the specific case from room temperature 
deformation of aluminium considered here the following analysis assumes that

- The GNB lies on the exact slip plane, i.e. $\mathbf{n}=(111)$.

- All boundary dislocations come from the four slip systems expected active, i.e. only the Burgers vectors b1, b2, b4 and b5 and slip planes (111) and (111) are considered.

- The dislocations only move by glide in their slip plane.

The first assumption about the boundary plane is introduced to simplify the analysis. The small deviations from the ideal (111) plane exhibited by real GNBs are considered when comparing with the experimental data and the implications of the deviations are treated in the discussion.

\subsection{Solving the Frank equation}

A boundary fulfilling Equation (1) with only one set of dislocations is a tilt boundary on a plane perpendicular to the Burgers vector [37]. This is impossible for $n=(111)$ in the fcc system.

The mathematical procedure to solve Equation (1) for two sets of dislocations with Burgers vectors $\mathbf{b} \boldsymbol{x}$ and by restricted to motion by glide on the planes $\mathbf{p} \boldsymbol{x}$ and $\mathbf{p} \boldsymbol{y}$, respectively, is given in [37] and also employed by others, e.g. [27,33,38,39]:

$$
\begin{gathered}
\mathbf{a} \| \mathbf{b} x \times \mathbf{b} y \\
\mathbf{n} \|\{(\mathbf{p} x \times \mathbf{a}) \times \mathbf{p} y\} \times\{(\mathbf{p} y \times \mathbf{a}) \times \mathbf{p} x\} \\
\mathbf{N} x=\frac{\mathbf{b} y-\mathbf{n}(\mathbf{n} \cdot \mathbf{b} y)}{|\mathbf{b} \boldsymbol{x} \times \mathbf{b} y|} ; \mathbf{N} y=\frac{-\mathbf{b} x-\mathbf{n}(\mathbf{n} \cdot \mathbf{b} x)}{|\mathbf{b} x \times \mathbf{b} y|} \\
\xi x=\mathbf{N} x \times \mathbf{n} ; \xi y=\mathbf{N} y \times \mathbf{n} \\
\rho x=|\mathbf{N} x| ; \rho y=|\mathbf{N} y|
\end{gathered}
$$

$\mathbf{N} \boldsymbol{x}$ and $\mathbf{N} \boldsymbol{y}$ are auxiliary vectors used to calculate the dislocation lines $\boldsymbol{\xi} \boldsymbol{x}$ and $\boldsymbol{\xi} \boldsymbol{y}$ and the dislocation densities $\rho x$ and $\rho y$. The flow of the calculations follows the order of the sub-equations in Eq. (2).

For three or four dislocations coming from two different glide planes, $\mathbf{p} x=(111)$ and $\mathbf{p} \boldsymbol{y}=(11 \overline{1})$, resulting Burgers vectors, $\mathbf{b} \boldsymbol{x}$ and $\mathbf{b y}$, for each slip plane are defined. The quantities $f x$ and $f y$ define the relative densities and signs of the two dislocations gliding on the same slip plane:

$$
\begin{gathered}
\mathbf{b} \boldsymbol{x}=f x \cdot \mathbf{b} 1+(1-f x) \cdot \mathbf{b} 2 ; \mathbf{b} \boldsymbol{y}=f y \cdot \mathbf{b} 4+(1-f y) \cdot \mathbf{b} 5 ;-1 \leq f x, f y \leq 1 ; \\
\rho 1=|f x||\mathbf{N} \boldsymbol{x}| ; \rho 2=(1-f x)|\mathbf{N} \boldsymbol{x}| ; \rho 4=|f y||\mathbf{N} \boldsymbol{y}| ; \rho 5=(1-f y)|\mathbf{N} \boldsymbol{y}|
\end{gathered}
$$

These equations are substituted into Eq. (2). Insertion of the present Burgers vectors and slip planes 
yields:

$$
\begin{gathered}
\mathbf{a} \|\left[\begin{array}{c}
1.5-f y \\
-0.5-f y \\
0.5-f y
\end{array}\right] \\
\text { and } \\
\mathbf{n} \|\left[\begin{array}{c}
3 f x-4 f x f y+3 f y-2 \\
-f x+4 f x f y-f y \\
-f x+f y
\end{array}\right]
\end{gathered}
$$

For $\mathbf{n}=(111)$ all three components of $\mathbf{n}$ must be equal. Equating each of the first two rows in $\mathbf{n}$ to the third one leads upon reduction to the following two equations:

$$
2 f x-1=0 \text { and } f y(1-2 f x)=0,
$$

which are fulfilled for $f x=0.5$ and any value of $f y$, except for a singularity for $f y=0.5$ where all components of $\mathbf{n}$ become 0 . This means that $\mathbf{b} 1$ and $\mathbf{b} 2$ must be present in equal densities, whereas the relative densities of $\mathbf{b 4}$ and $\mathbf{b 5}$ may vary freely.

As an alternative to Eq. (2), the boundary may be a boundary with the misorientation axis [37]

$$
\text { a \| } \mathbf{p} x \times \mathbf{p} y,
$$

A symmetric tilt boundary with $a=\left[\begin{array}{lll}1 & 1 & 0\end{array}\right]$ defines the boundary network for fy=0.5.

\subsection{Dislocation lines}

Due to the assumption of dislocation motion by glide only $\boldsymbol{\xi} \boldsymbol{y}=\boldsymbol{\xi} \mathbf{4}=\boldsymbol{\xi} \mathbf{5}$ is always parallel to [1 110$]$, which is the intersection line between $\mathbf{p y}$ and $\mathbf{n}$. As the dislocations b1 and $\mathbf{b 2}$ may glide in the boundary plane they have some freedom with respect to the dislocation lines: while the resultant dislocation line $\xi \boldsymbol{x}$ is given by Equation (3), the directions of $\boldsymbol{\xi} \mathbf{1}$ and $\boldsymbol{\xi} 2$ may vary. For a boundary fulfilling the Frank equation with alternative dislocation lines $\boldsymbol{\xi} \mathbf{1}$ and $\boldsymbol{\xi} \mathbf{2}$ the following equation must hold:

$$
\rho 1^{\prime} \mathbf{b} 1\{(\mathbf{n} \times \xi \mathbf{1}) \cdot \mathbf{V}\}+\rho 2^{\prime} \mathbf{b} 2\{(\mathbf{n} \times \xi \mathbf{2}) \cdot \mathbf{V}\}=(\rho 1 \mathbf{b 1}+\rho 2 \mathbf{b 2})\{(\mathbf{n} \times \xi \mathbf{x}) \cdot \mathbf{V}\},
$$

where $\rho 1^{\prime}$ and $\rho 2^{\prime}$ are the densities in the alternative configuration. Using the relation $(\mathbf{n} \times \boldsymbol{\xi}) \cdot \mathbf{V}=\boldsymbol{\xi}$. $(\mathbf{V} \times \mathbf{n})$ and setting $\mathbf{R}=\mathbf{V} \times \mathbf{n}$, this transforms to 


$$
\rho 1^{\prime} \mathbf{b} 1(\xi \mathbf{1} \cdot \mathbf{R})+\rho 2^{\prime} \mathbf{b} 2(\xi 2 \cdot \mathbf{R})=(\rho 1 \mathbf{b} \mathbf{1}+\rho 2 \mathbf{b} 2)(\xi \boldsymbol{x} \cdot \mathbf{R}) .
$$

By insertion of the known Burgers vectors $\mathbf{b 1}$ and $\mathbf{b} 2$ as well as the calculated $\boldsymbol{\xi} \boldsymbol{x} \|[f y$; fy$1 ; 1-2 f y]$ and exploiting the fact that $\mathbf{R}, \boldsymbol{\xi} \mathbf{1}$ and $\xi \mathbf{2}$ are vectors in the boundary plane, i.e. perpendicular to $\mathbf{n}$, it can be shown that the only solution is for $\boldsymbol{\xi} \mathbf{1}=\boldsymbol{\xi} \mathbf{2}=\boldsymbol{\xi} \boldsymbol{x}$.

\subsection{Dislocation reactions}

Dislocation reactions in the boundary giving dislocations of other Burgers vectors may further lower the total elastic energy of the dislocations. According to the so-called Frank rule [37] reaction between dislocations with Burgers vectors ba and $\mathbf{b b}$ to form $\mathbf{b c}$ is energetically favourable if $|\mathbf{b c}|^{2}<|\mathbf{b a}|^{2}+|\mathbf{b b}|^{2}$. In the present case this enables the following reactions:

$$
\begin{aligned}
& \mathbf{b} 1-\mathbf{b} 2=\left[\begin{array}{lll}
1 & 0 & \overline{1}
\end{array}\right]-\left[\begin{array}{lll}
0 & 1 & \overline{1}
\end{array}\right]=\left[\begin{array}{lll}
1 & \overline{1} & 0
\end{array}\right]=\mathbf{b 3} ; \\
& \mathbf{b} 1+\mathbf{b} 5=\left[\begin{array}{lll}
1 & 0 & \overline{1}
\end{array}\right]+\left[\begin{array}{lll}
0 & 1 & 1
\end{array}\right]=\left[\begin{array}{lll}
1 & 1 & 0
\end{array}\right]=\mathbf{b} 6 ; \\
& \mathbf{b 2}+\mathbf{b} 4=\left[\begin{array}{lll}
0 & 1 & \overline{1}
\end{array}\right]+\left[\begin{array}{lll}
1 & 0 & 1
\end{array}\right]=\left[\begin{array}{lll}
1 & 1 & 0
\end{array}\right]=\mathbf{b} 6 .
\end{aligned}
$$

In order for $\mathbf{b 6}$ to form, dislocations from two slip planes must meet, i.e. the intersection line between the two slip planes becomes parallel to $\boldsymbol{\xi} \mathbf{6}$. The result is a sessile Lomer lock. By contrast dislocations $\mathbf{b} \mathbf{1}$ and $\mathbf{b} 2$ may meet anywhere in their common glide plane to form $\mathbf{b 3}$.

Introduction of dislocation reactions in the Frank equation (Equation (1)) has consequences for the dislocation lines and densities of the involved dislocations. The contributions to the equation must be the same before and after reaction:

$$
\begin{gathered}
\left(\rho a-\rho c^{\prime}\right) \mathbf{b a}\left(\xi \boldsymbol{a}^{\prime} \cdot \mathbf{R}\right)+\left(\rho b-\rho c^{\prime}\right) \mathbf{b b}\left(\xi \boldsymbol{b}^{\prime} \cdot \mathbf{R}\right)+\rho c^{\prime} \mathbf{b c}\left(\xi \boldsymbol{c}^{\prime} \cdot \mathbf{R}\right) \\
=\rho a \mathbf{b a}(\xi \boldsymbol{a} \cdot \mathbf{R})+\rho b \mathbf{b b}(\xi \boldsymbol{b} \cdot \mathbf{R}),
\end{gathered}
$$

where quantities marked ' refer to the configuration after reaction whereas those without this mark represent the initial unreacted condition. The dislocation lines $\boldsymbol{\xi} \mathbf{4}, \boldsymbol{\xi} \mathbf{5}$ and $\boldsymbol{\xi} \mathbf{6}$ are fixed for reasons described above. By contrast $\boldsymbol{\xi} \mathbf{1}, \boldsymbol{\xi} \mathbf{2}$ and $\boldsymbol{\xi} \mathbf{3}$ may vary.

\section{Results}

This section presents the dislocation networks corresponding to the solutions to the Frank equation derived in section 2. For clarity all vectors are written using integral indices but in the calculations the proper normalisation has been applied.

\subsection{Two sets of dislocations}

In the present case the only solution with two dislocations for $\mathbf{n}=(111)$ is for $\mathbf{b} \mathbf{1}$ and $\mathbf{b} \mathbf{2}$, both gliding in 
(111), i.e. $\mathrm{px}=\mathrm{py}=(111)$. It follows from Equation (2) that two sets of dislocations coming from the slip plane (111) with which the GNB aligns (i.e. b1 and b2) can produce a twist boundary. The dislocation content is given by Equation (11).

$$
\begin{aligned}
& \mathbf{n}=\left(\begin{array}{lll}
1 & 1 & 1
\end{array}\right) ; \mathbf{a}=\left[\begin{array}{lll}
1 & 1 & 1
\end{array}\right] ; \mathbf{b} 1=\left[\begin{array}{lll}
1 & 0 & \overline{1}
\end{array}\right] ; \mathbf{b} 2=\left[\begin{array}{lll}
0 & 1 & \overline{1}
\end{array}\right] \\
& \boldsymbol{\xi} \mathbf{1}=\left[\begin{array}{lll}
2 & \overline{1} & \overline{1}
\end{array}\right] ; \quad \xi 2=\left[\begin{array}{lll}
\overline{1} & 2 & \overline{1}
\end{array}\right] \\
& \rho 1=\rho 2
\end{aligned}
$$

Dislocation reaction in the boundary between $\mathbf{b 1}$ and $\mathbf{b 2}$ to produce $\mathbf{b 3}$ and the resulting changes to dislocation lines and densities are calculated by means of Equation (10). For an arbitrary set of $\xi \mathbf{3}^{\prime}$ and $\rho 3$ Equation (10) and Equation (11) lead to the following relationships:

$$
\begin{gathered}
\boldsymbol{\xi} \mathbf{1}^{\prime}\left\|\rho 1 \boldsymbol{1}-\rho 3^{\prime} \boldsymbol{\xi} \mathbf{3}^{\prime} ; \boldsymbol{\xi} \mathbf{2}^{\prime}\right\| \rho 1 \xi 2+\rho 3^{\prime} \boldsymbol{\xi} \mathbf{3}^{\prime} ; \\
\rho 1^{\prime}=\left(\rho 1-\rho 3^{\prime}\right)\left|\rho 1 \xi \mathbf{1}-\rho 3^{\prime} \boldsymbol{\xi} \mathbf{3}^{\prime}\right| ; \rho 2^{\prime}=\left(\rho 1-\rho 3^{\prime}\right)\left|\rho 1 \xi 2+\rho 3^{\prime} \boldsymbol{\xi} \mathbf{3}^{\prime}\right|
\end{gathered}
$$

From Equation (12) it follows that combination of the entire populations of $\mathbf{b} \mathbf{1}$ and $\mathbf{b 2}$ into $\mathbf{b 3}$ is not in agreement with the Frank equation. The smallest total dislocation density in the boundary is obtained for:

$$
\begin{aligned}
& \mathbf{n}=\left(\begin{array}{lll}
1 & 1 & 1
\end{array}\right) ; \mathbf{a}=\left[\begin{array}{lll}
1 & 1 & 1
\end{array}\right] ; \mathbf{b} \mathbf{1}=\left[\begin{array}{lll}
1 & 0 & \overline{1}
\end{array}\right] ; \mathbf{b} 2=\left[\begin{array}{lll}
0 & 1 & \overline{1}
\end{array}\right] ; \mathbf{b 3}=\left[\begin{array}{lll}
1 & \overline{1} & 0
\end{array}\right] \\
& \xi \mathbf{1}=\left[\begin{array}{lll}
1 & 0 & \overline{1}
\end{array}\right] ; \boldsymbol{\xi} \mathbf{2}=\left[\begin{array}{lll}
0 & 1 & \overline{1}
\end{array}\right] ; \xi 3=\left[\begin{array}{lll}
1 & \overline{1} & 0
\end{array}\right] \\
& \rho 1=\rho 2=\rho 3
\end{aligned}
$$

In Equation (13) the total dislocation density is a factor of $\sqrt{3}$ smaller than in Equation (11) for the same misorientation angle $\theta$. This network is illustrated in Figure 2. This hexagonal network further has equilibrium angles of $120^{\circ}$ at the dislocation nodes and are therefore expected to be the most energetically favourable configuration. This theoretical configuration is also well-known in the literature [37]. 


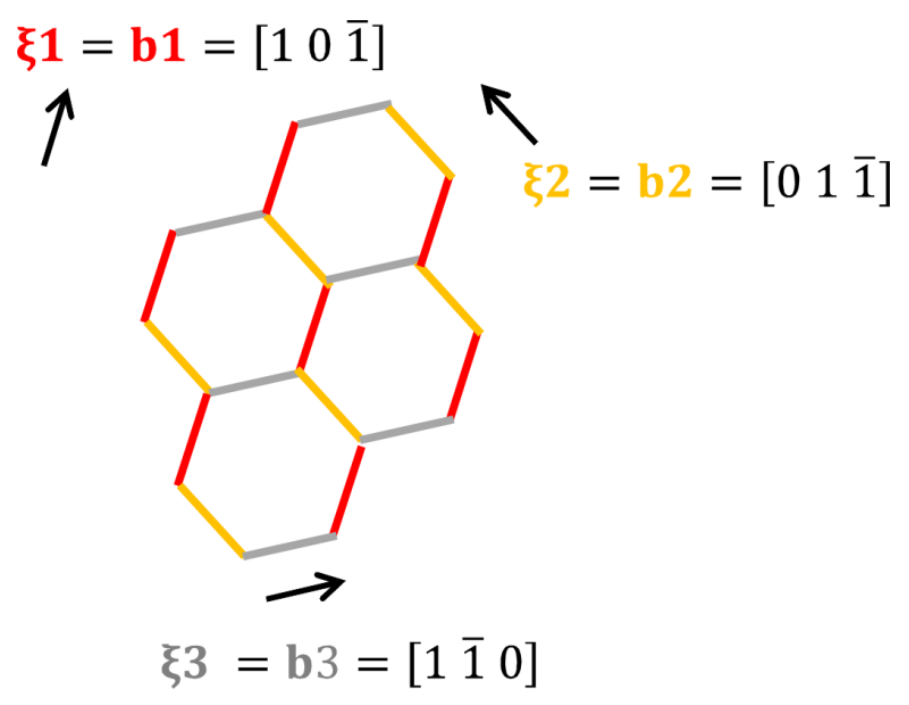

Figure 2. Dislocation network on (111) constructed from two sets of dislocations from the (111) slip plane after dislocation reactions in the boundary.

\subsection{Three sets of dislocations.}

With three sets of dislocations two basic configurations are possible according to Equations (2)-(5). In both of these, the two sets of dislocations gliding on planes parallel to the GNB, i.e. b1 and $\mathbf{b 2}$, are present in equal densities $(f x=0.5)$ while either $\mathbf{b 4}(f y=1)$ or $\mathbf{b 5}(f y=0)$ comes from the other slip plane. The dislocation lines of $\mathbf{b 1}$ and $\mathbf{b 2}$ are parallel as described in Equations (14) and (15). The two configurations have different misorientation axes but both have mixed tilt/twist character and they are essentially symmetric.

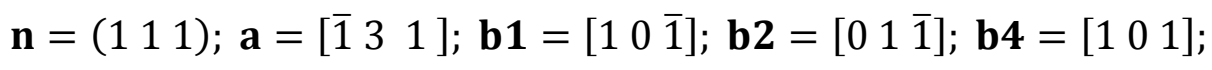

$$
\begin{aligned}
& \boldsymbol{\xi 1}=\boldsymbol{\xi 2}=\left[\begin{array}{lll}
1 & 0 & \overline{1}
\end{array}\right] ; \boldsymbol{\xi} \mathbf{4}=\left[\begin{array}{lll}
1 & \overline{1} & 0
\end{array}\right] \\
& \rho 1=\rho 2=\rho 4 / 3
\end{aligned}
$$

and

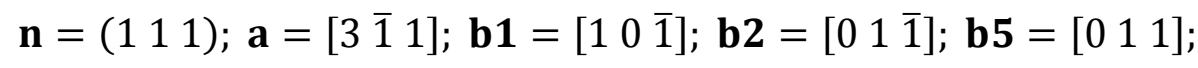

$$
\begin{aligned}
& \boldsymbol{\xi} \mathbf{1}=\boldsymbol{\xi} \mathbf{2}=\left[\begin{array}{lll}
0 & 1 & \overline{1}
\end{array}\right] ; \boldsymbol{\xi} \mathbf{5}=\left[\begin{array}{lll}
\overline{1} & 1 & 0
\end{array}\right] ;
\end{aligned}
$$




$$
\rho 1=\rho 2=\rho 5 / 3
$$

Dislocation reactions between $\mathbf{b 2}$ and $\mathbf{b 4}$ or between b1 and $\mathbf{b 5}$ may lead to formation of Lomer locks as described in section 2. Dislocation networks containing such Lomer locks and still fulfilling the Frank equation are obtained by modification of Equations (14)-(15) according to Equation (10) with bc=b6. The indices $a$ and $b$ are 2 and 4 for Equation (14) and 1 and 5 for Equation (15). This results in the following relations:

$$
\begin{gathered}
\xi \mathbf{a}^{\prime} \| \rho a \xi \mathbf{a}-\rho 6^{\prime} \boldsymbol{\xi} \mathbf{b} ; \\
\rho \mathbf{a}^{\prime}=\left|\rho \mathbf{a} \xi \mathbf{a}-\rho 6^{\prime} \xi \mathbf{b}\right| ; \rho \mathbf{b}^{\prime}=\left(\rho b-\rho 6^{\prime}\right)
\end{gathered}
$$

Also in this case consumption of the entire population of $\mathbf{b} \mathbf{1}$ by this reaction is not possible. Equations (17)-(18), where both $\mathbf{b} 1$ and $\mathbf{b 2}$ are screws, define networks where the total dislocation density is the same as in Equations (14)-(15) for the same misorientation angle $\theta$. Figure 3 illustrates these new boundary networks.

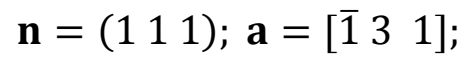

$$
\begin{aligned}
& \mathbf{b 1}=\left[\begin{array}{lll}
1 & 0 & \overline{1}
\end{array}\right] ; \mathbf{b} 2=\left[\begin{array}{lll}
0 & 1 & \overline{1}
\end{array}\right] ; \mathbf{b} 4=\left[\begin{array}{lll}
1 & 0 & 1
\end{array}\right] ; \mathbf{b} 6=\left[\begin{array}{lll}
1 & 1 & 0
\end{array}\right] \text {; } \\
& \boldsymbol{\xi 1}=\left[\begin{array}{lll}
1 & 0 & \overline{1}
\end{array}\right] ; \boldsymbol{\xi} \mathbf{2}=\left[\begin{array}{lll}
0 & 1 & \overline{1}
\end{array}\right] ; \boldsymbol{\xi} \mathbf{4}=\boldsymbol{\xi} \mathbf{6}=\left[\begin{array}{lll}
1 & \overline{1} & 0
\end{array}\right] ; \\
& \rho 1=\rho 2=\rho 6=\rho 4 / 2
\end{aligned}
$$

and

$$
\begin{aligned}
& \mathbf{n}=\left(\begin{array}{lll}
1 & 1 & 1
\end{array}\right) ; \mathbf{a}=\left[\begin{array}{lll}
3 & \overline{1} & 1
\end{array}\right] ; \\
& \mathbf{b 1}=\left[\begin{array}{lll}
1 & 0 & \overline{1}
\end{array}\right] ; \mathbf{b} 2=\left[\begin{array}{lll}
0 & 1 & \overline{1}
\end{array}\right] ; \mathbf{b} 5=\left[\begin{array}{lll}
0 & 1 & 1
\end{array}\right] ; \mathbf{b} 6=\left[\begin{array}{lll}
1 & 1 & 0
\end{array}\right] ; \\
& \boldsymbol{\xi} \mathbf{1}=\left[\begin{array}{lll}
1 & 0 & \overline{1}
\end{array}\right] ; \boldsymbol{\xi} \mathbf{2}=\left[\begin{array}{lll}
0 & 1 & \overline{1}
\end{array}\right] ; \xi \mathbf{5}=\boldsymbol{\xi} \mathbf{6}=\left[\begin{array}{lll}
\overline{1} & 1 & 0
\end{array}\right] ; \\
& \rho 1=\rho 2=\rho 6=\rho 5 / 2
\end{aligned}
$$

It should be noted that a configuration with $\xi \mathbf{a}^{\prime}$ parallel to $[11 \overline{2}]$ minimises the total dislocation 
density compared to Equations (17)-(18) and Figure 3.
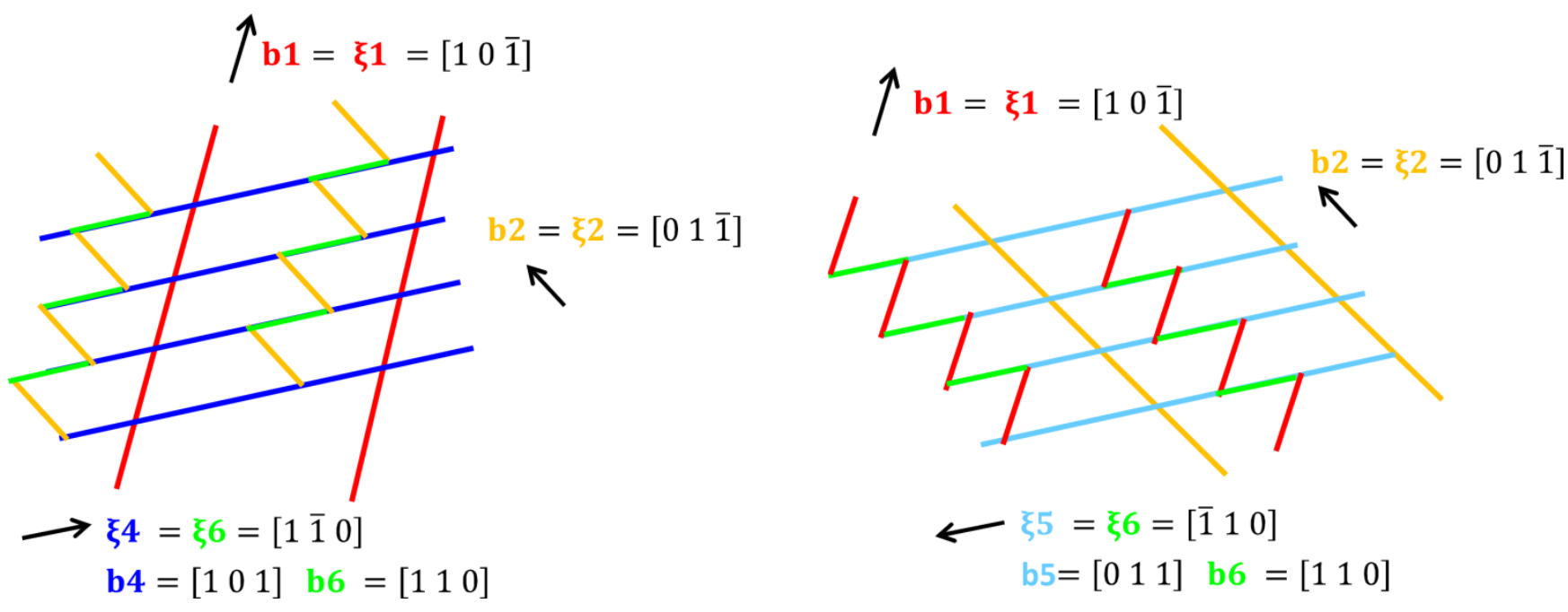

Figure 3. Dislocation networks on (111) constructed from three sets of dislocations; $\boldsymbol{b} 1$ and $\boldsymbol{b} 2$ from (111); and from (11̄1) b4 (left) or b5 (right) after reaction to form Lomer locks of b6.

\subsection{Four sets of dislocations}

Application of Equations (2)-(5) with $-1<f y<1$ essentially results in solutions to the Frank equation that are linear combinations of those in Equations (14)-(15), which after reactions to form Lomer locks transforms into linear combinations of Equations (17)-(18). In some of the linear combinations dislocations with the same Burgers vector but opposite signs of the dislocation line will be present as redundant dislocations. This may of course annihilate.

A network corresponding to a twist boundary with both b1 and b2 as screws and equal densities of b4 and $\mathbf{b 5}$ is obtained by addition of Equations (17)-(18):

$$
\begin{gathered}
\mathbf{n}=\left(\begin{array}{lll}
1 & 1 & 1
\end{array}\right) ; \mathbf{a}=\left[\begin{array}{lll}
1 & 1 & 1
\end{array}\right] ; \\
\mathbf{b 1}=\left[\begin{array}{lll}
1 & 0 & \overline{1}
\end{array}\right] ; \mathbf{b} 2=\left[\begin{array}{lll}
0 & 1 & \overline{1}
\end{array}\right] ; \mathbf{b} 4=\left[\begin{array}{lll}
1 & 0 & 1
\end{array}\right] ; \mathbf{b 5}=\left[\begin{array}{lll}
0 & 1 & 1
\end{array}\right] ; ; \\
\boldsymbol{\xi 1}=\left[\begin{array}{lll}
1 & 0 & \overline{1}
\end{array}\right] ; \xi 2=\left[\begin{array}{lll}
0 & 1 & \overline{1}
\end{array}\right] ; \xi 4=-\xi 5=\left[\begin{array}{lll}
\overline{1} & 1 & 0
\end{array}\right] ; \\
\rho 1=\rho 2=\rho 4 / 2=\rho 5 / 2
\end{gathered}
$$


This network may contain positive and negative Lomer locks of equal densities as redundant dislocations, which are produced by local reactions, as illustrated in the left part of Figure 4, which is a superposition of the two networks in Figure 3.

It is noteworthy that the symmetric tilt boundary obtained from Equation (6) is obtained by subtraction of Equations (17)-(18). Equation (6) yields a network with b1, b2, b4 and b5 where all the dislocation lines are parallel. By contrast to the previous cases, the entire populations of $\mathbf{b 1}$ and $\mathbf{b 2}$ may react with the two others to produce b6. This results in the following tilt boundary, which is also illustrated in the right part of Figure 4:

$$
\begin{gathered}
\mathbf{n}=\left(\begin{array}{lll}
1 & 1 & 1
\end{array}\right) ; \mathbf{a}=\left[\begin{array}{lll}
\overline{1} & 1 & 0
\end{array}\right] ; \\
\mathbf{b 4}=\left[\begin{array}{lll}
1 & 0 & 1
\end{array}\right] ; \mathbf{b} \mathbf{5}=\left[\begin{array}{lll}
0 & 1 & 1
\end{array}\right] ; \mathbf{b} \mathbf{6}=\left[\begin{array}{lll}
1 & 1 & 0
\end{array}\right] ; \\
\xi 4=\xi 5=\xi 6=\left[\begin{array}{lll}
\overline{1} & 1 & 0
\end{array}\right] ; \\
\rho 4=\rho 5=\rho 6
\end{gathered}
$$

The dislocations $\mathbf{b 1}$ and $\mathbf{b 2}$ may, however, also react with segments from different dislocations, leading to interconnecting segments of $\mathbf{b 1}$ and $\mathbf{b 2}$ in the final network. Such segments will also be present in the case of imbalance in the contributions from Equations (17)-(18).
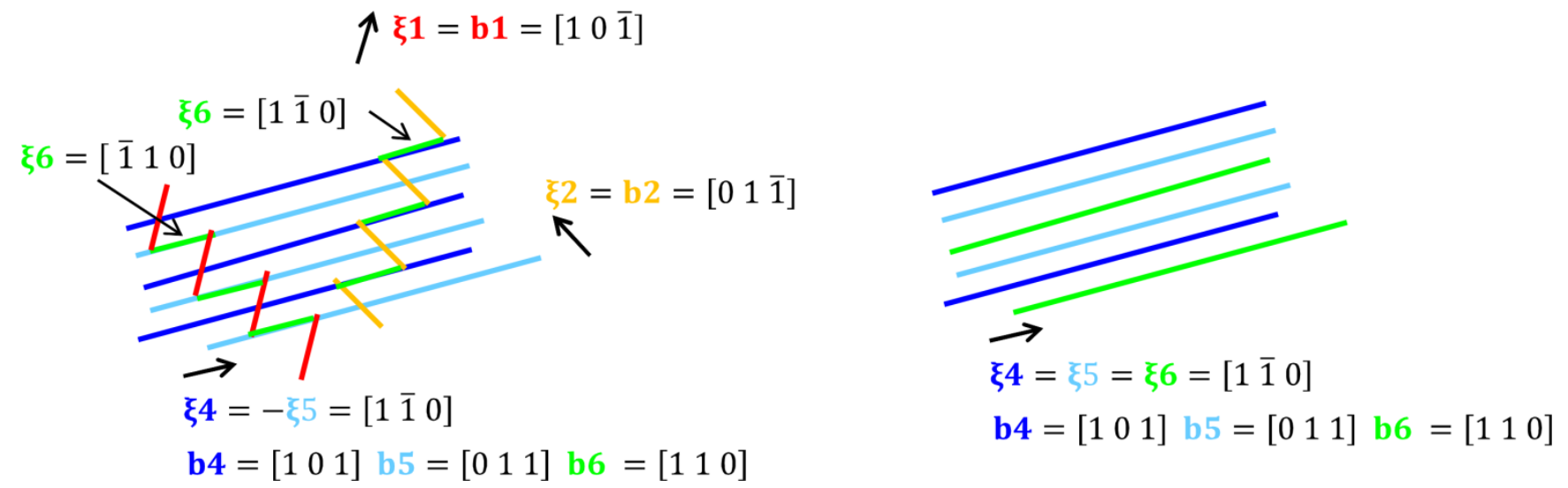

Figure 4. Dislocation networks on (111) consisting of five sets of dislocations, of which b6 is a Lomer lock. Left: twist boundary (Equation (19)). Right: tilt boundary ((Equation (20)).

In summary, all dislocation networks in boundaries aligned with (111) which fulfil the Frank equation with the gliding dislocations considered here have been identified, allowing for reactions between dislocations. They are described mathematically in terms of the presented equations or are linear combinations of these equations. The resulting theoretical span of misorientation axes is illustrated in Figure 5 and constitutes an arc in the inverse pole figure. Graphical illustrations of representative networks are presented in Figures 2-4. 


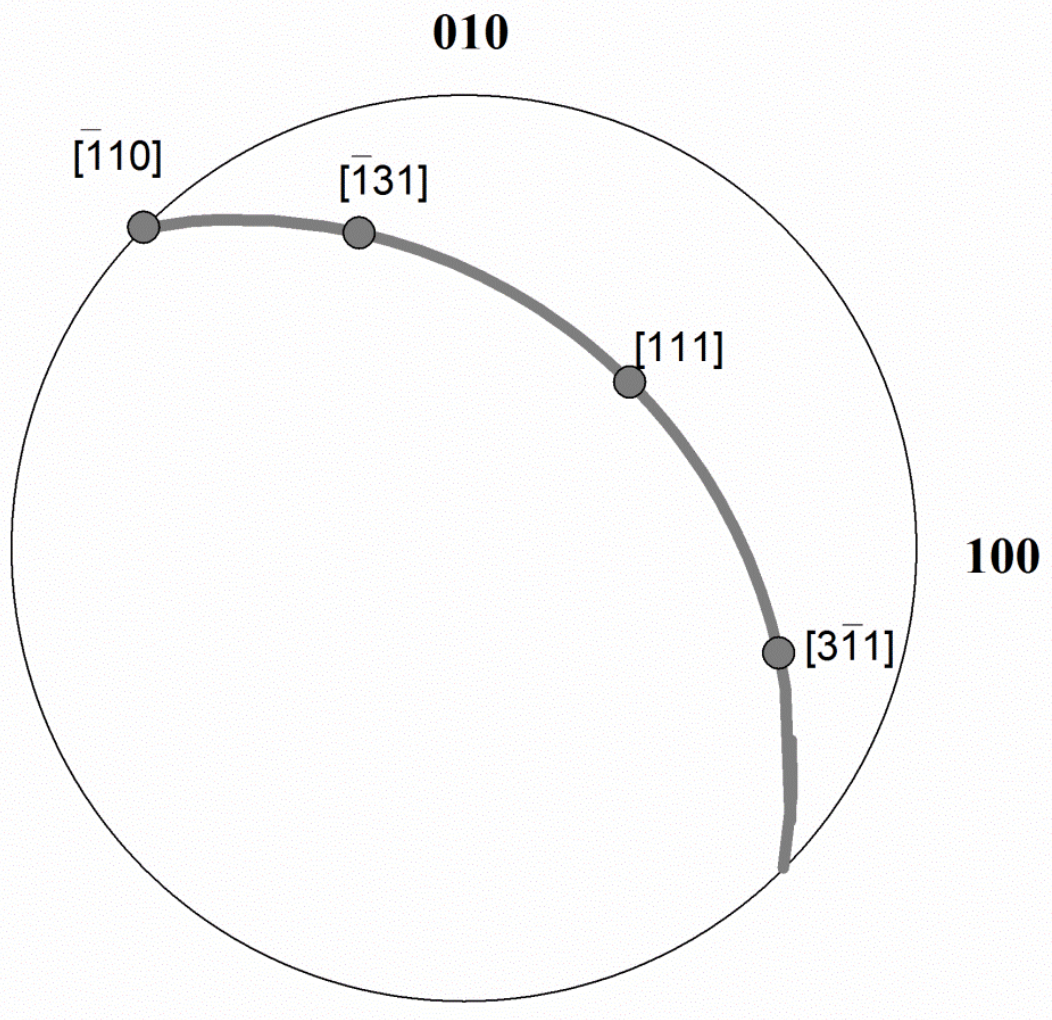

Figure 5. Inverse pole figure showing the theoretically predicted misorientation axes from Equations (11) to (18) as circles. The arc connecting them is the theoretically possible span of misorientation axes produced by linear combinations.

\section{Comparison with experiment}

\subsection{Summary of experimental observations}

The experimentally observed dislocation networks of eight slip-plane-aligned GNBs in three grains of the $45^{\circ} \mathrm{ND}$ rotated Cube orientation after $10 \%$ rolling of high purity aluminium presented in a previous paper [31] are shown in Figure 6 to facilitate the comparison with the present theoretical predictions. The experimental networks fell in two main categories:

- Three sets of dislocations in a hexagonal network, all having Burgers vectors in the slip plane with which the boundary aligned. Two such GNBs (GNB1 and 2) were observed, both aligned with (111) (see Figure 6a). 
- Dislocations coming from both of the active slip planes and an additional set believed to be Lomer locks.

- Figure $6 \mathrm{~b}$ shows GNB3 and 7, which both align with (111), and almost exclusively contain $\mathrm{b5}$ and $\mathrm{b4}$, respectively, in addition to $\mathrm{b} 1$ and $\mathrm{b} 2$ gliding in the boundary plane.

- Figure 6c shows GNB4 aligned with (111) with all four sets of dislocations.

- Figure 6d shows GNBs 5, 6 and 8 aligned with the other slip plane, i.e. (111) $)$, with $\mathbf{b 4}$ and $\mathbf{b 5}$ gliding in the boundary plane and $\mathbf{b 2}$ coming from the other slip plane. 


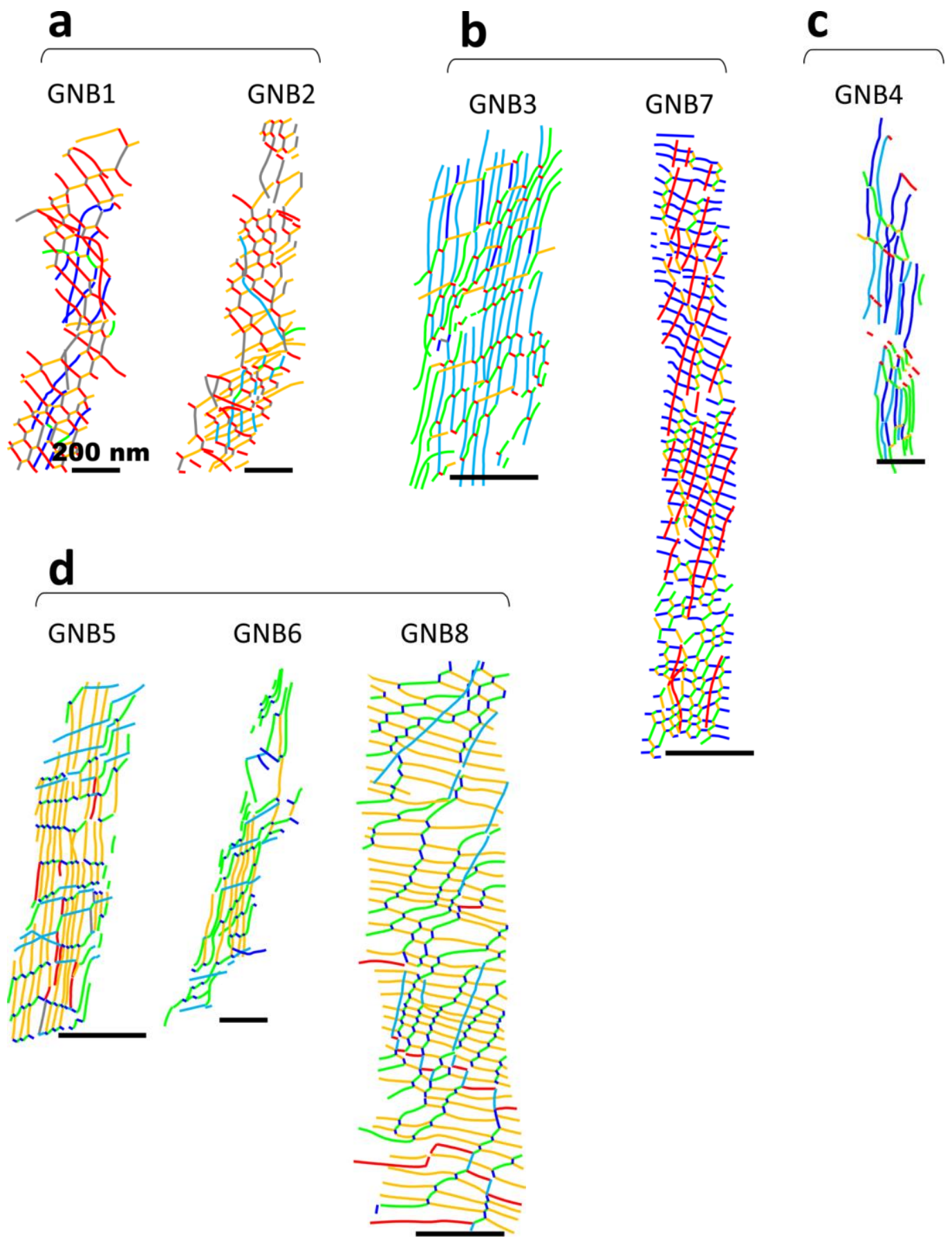

Figure 6. The experimentally determined dislocation content of GNB1-8 from [31]. GNBs 5, 6 and 8 align with (11 $\overline{1})$ and the rest with (111). See text for a further description of the groups a-d. 
GNB2
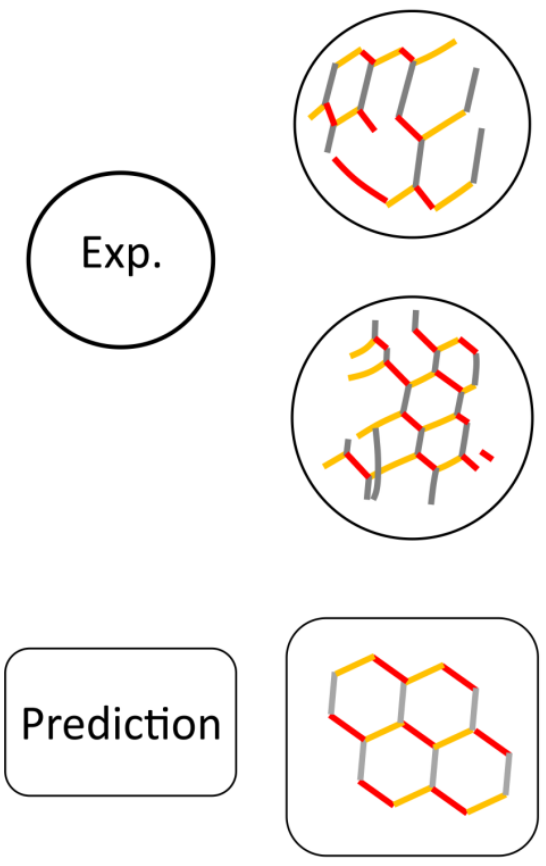

GNB7
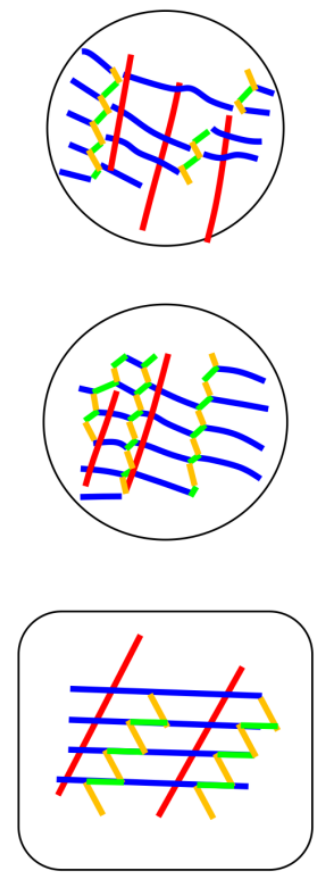

GNB3
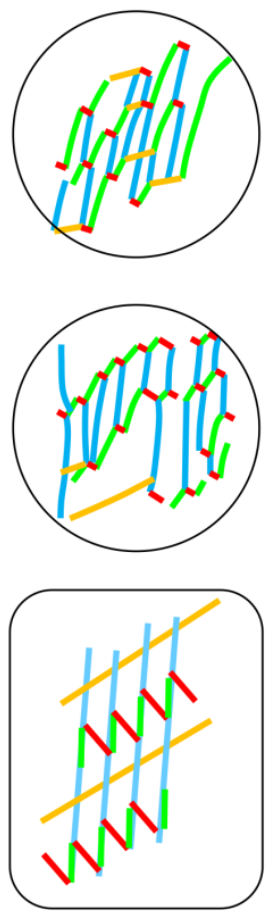

GNB4
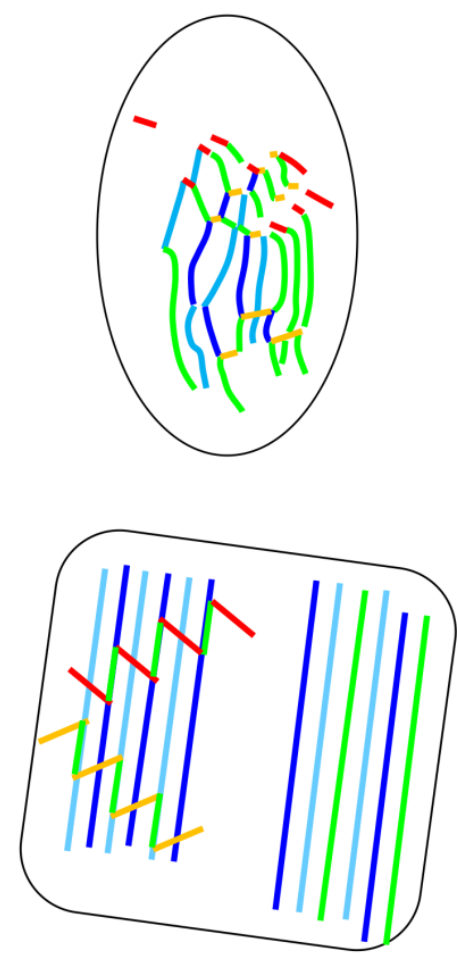

Figure 7. Representative enlarged segments of the experimental dislocation networks are shown inside the circles whereas the boxes below show the idealised predicted networks from Figure 2, Figure 3 and Figure 4.

\subsection{Qualitative comparison}

Figure 7 is a qualitative comparison of representative enlarged segments of the dislocation networks aligned with (111) from Figure 6 with the corresponding theoretical predictions from Figure 2, Figure 3 and Figure 4. The experimental segments of GNB2 consist of the three dislocations b1, b2 and b3 gliding in the (111) slip plane with which the boundary aligns. The morphology of the network is obviously in good agreement with the theoretically predicted regular hexagonal network and all three sets of dislocations have strong screw character as determined in the experimental paper [31] as well as by Equation (3).

The networks from GNB7 strongly resemble the theoretical networks in the left part of Figure 3 constructed from b1 and b2 gliding in (111) and b4 in (11 $\overline{1})$. The segments from GNB3 are in corresponding good agreement with the right part of Figure 3, where b5 is included instead of b4. All networks have Lomer locks (b6) present as reaction products, in agreement with the predictions. Note 
that dislocations of the same Burgers vector are parallel to each other, both in the experimental and theoretical networks. Comparison of the experimental (see [31]) and predicted dislocation line directions also reveals good agreement: $\mathbf{b 1}$ and $\mathbf{b 2}$ have strong screw character while the dislocation lines of $\mathbf{b 4 , ~} \mathbf{b 5}$ and $\mathbf{b 6}$ are roughly parallel to the intersection line between the boundary plane and the

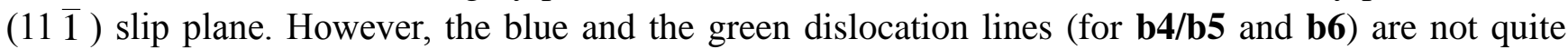
parallel in the experimental networks by contrast to the prediction. By nature a Lomer lock is parallel to the intersection between two slip planes. As the experimentally observed boundary planes deviate a few degrees from the exact $\{111\}$, this dislocation line is not identical to the intersection between the slip plane of b4/b5 and the boundary plane, which defines the dislocation lines $\boldsymbol{\xi 4 / \xi 5}$. Consequently the blue and green dislocation lines will not be parallel for real GNBs. This implies that the Lomer locks are not lying flat in the boundary but stick out of this. The experimentally observed dislocation lines are the projections of the true lines onto the imaging plane in the TEM, which is close to the plane of the boundary in most cases. Projection effects also explain the minor deviations of the measured dislocation lines $\xi 6$ from [ $\left[\begin{array}{lll}\overline{1} & 1 & 0\end{array}\right]$ in the experimental paper [31].

The network of GNB4 is the most complex with all four sets of gliding dislocations as well as Lomer locks. Even in this case the experimentally determined dislocation lines are in agreement with the predictions from Figure 4: those of $\mathbf{b 4 , ~} \mathbf{b 5}$ and $\mathbf{b 6}$ are roughly parallel to the intersection line between the boundary plane and the (11 $\overline{1})$ slip plane and $\mathbf{b 1}$ and $\mathbf{b 2}$ have strong screw character. The low densities of b1 and $\mathbf{b 2}$ in this network as well as the high density of $\mathbf{b 6}$ suggest that this boundary is close to the tilt boundary in the right part of Figure 4.

Figure 8 shows the corresponding qualitative comparison of the network morphology for two representative segments of the GNBs aligned with the other slip plane, i.e. $\mathbf{n}=(11 \overline{1})$. The predicted network morphology is symmetrically equivalent to the ones in Figure 3, where only the dislocations on the two slip planes have been interchanged, obviously also with consequences for the misorientation axis as illustrated below. The agreement between experiment and prediction is as good as for the other GNBs. 

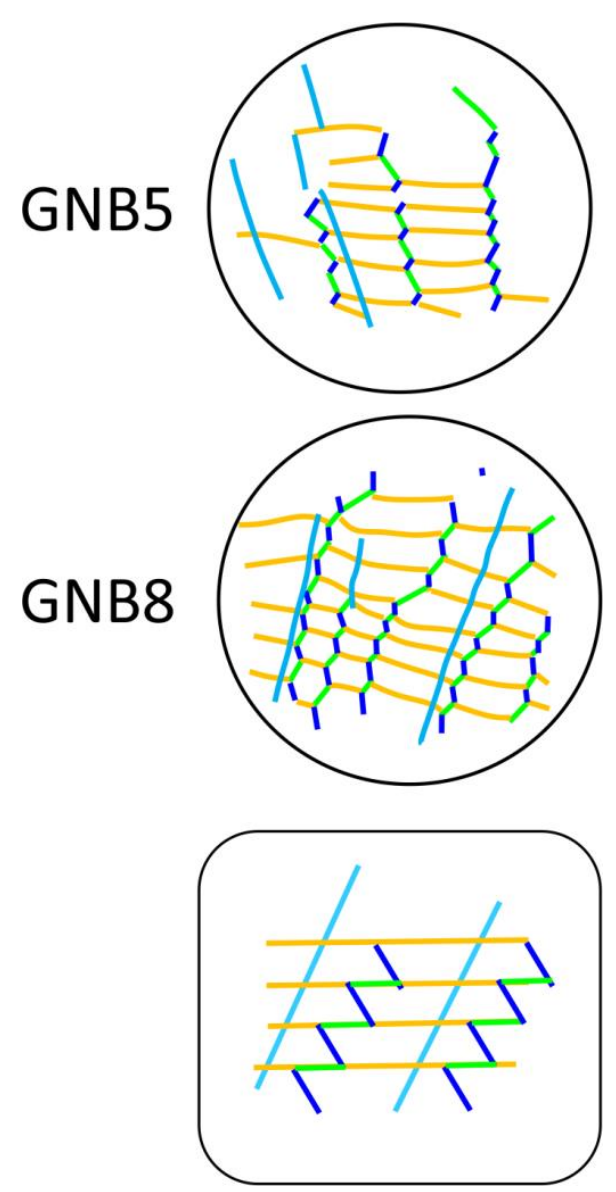

Figure 8. Comparison of experimental and predicted dislocation networks for a GNB segment representative of the boundaries aligned with (11) in the same way as in Figure 7.

\subsection{Quantitative comparison}

Quantitative evaluation of the agreement with the Frank equation - and therefore the LEDS character has been pursued based on evaluation of Equation (1) using experimentally measured values for all quantities. The two vectors representing the left and right hand sides of Equation (1) are termed B and $\mathrm{A}$ in the following, respectively, i.e.

$$
\mathrm{B}=\sum \rho_{\mathrm{i}} \mathbf{b}_{\mathbf{i}}\left\{\left(\mathbf{n} \times \boldsymbol{\xi}_{\mathbf{i}}\right) \cdot \mathbf{V}\right\}
$$

and

$$
\mathrm{A}=\mathbf{V} \times \mathbf{a}
$$


According to Equation (1) A and B should be parallel for all vectors $\mathbf{V}$ in the boundary plane. $\mathbf{V}_{\mathbf{1}}=\mathbf{b} \mathbf{1} \times \mathbf{n}$ is of course such a vector. By rotating this $\mathbf{V}$ in $10^{\circ}$ steps around the boundary normal $\mathbf{n}, \mathbf{A}$ and $\mathbf{B}$ were evaluated for eighteen $\mathbf{V}$-vectors, covering the entire boundary plane. For each $\mathbf{V}, \mathbf{A}$ and $\mathbf{B}$ were calculated from the measured $\mathbf{n}, \mathbf{b}_{\mathbf{i}}, \boldsymbol{\xi}_{\mathbf{i}}$ and $\rho_{\mathrm{i}}$ taken from [31]. The difference between $\mathbf{A}$ and $\mathbf{B}$ was then evaluated in terms of the angle between them averaged over the eighteen $\mathbf{V}$ vectors.

The vector A could only be evaluated directly for GNB7 and 8 as these two GNBs are the only ones for which the misorientation axes a have been measured in the TEM. Instead the misorientation axis a for each GNB has been estimated based on the realisation that this axis must be perpendicular to $\mathbf{A}$ (see Equation (22)) and therefore also perpendicular to B. Consequently a must be parallel to the cross product of two $\mathbf{B}$ vectors evaluated for two different vectors $\mathbf{V}$. The misorientation axis calculated in this way is independent of the directions of the two $\mathbf{V}$ vectors employed. As two segments of GNB7 were characterised experimentally the analysis has been conducted for a total of nine dislocation networks. The distribution of angles between A and B is presented in Figure 9, which shows that the deviation in general is about $10^{\circ}$.

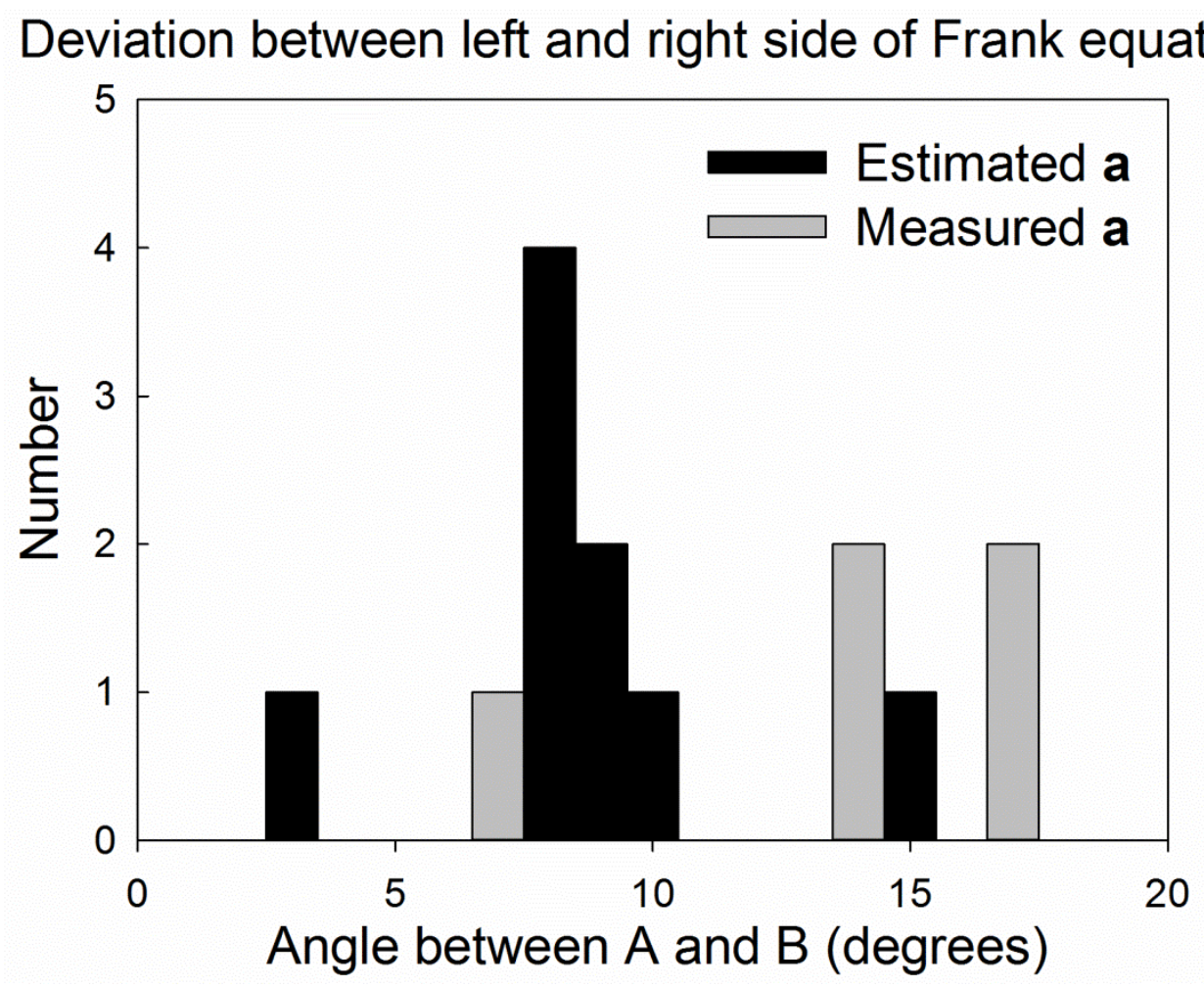

Figure 9. Comparison of the left and right hand sides of the Frank equation (Eq. (1)) in terms of the angle between the vectors $\boldsymbol{A}$ and $\boldsymbol{B}$ (see text). 

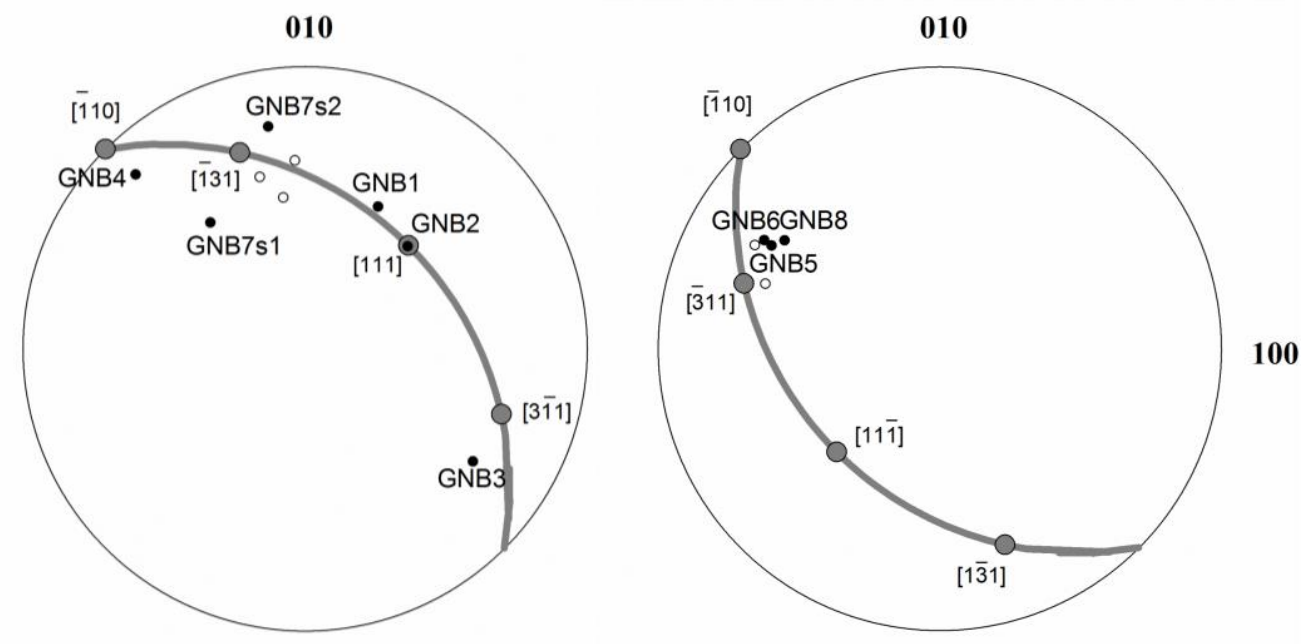

Figure 10. Comparison of misorientation axes based on experimental data (small black symbols) and the theoretically predicted span. Open symbols mark three independently measured experimental axes for GNB7 and two for GNB8. Solid symbols for all GNBs are axes calculated from the experimentally determined dislocation content and boundary plane. Left: GNBs aligned with (111); right GNBs aligned with (11 1 ). Solid grey symbols mark the predicted axes for basic configurations composed of two, three, and four sets of dislocations.

The misorientation axes themselves are plotted as black symbols in Figure 10 on top of the theoretical predictions (in grey). For two of the boundaries independent measurements of the axis (three for GNB7 and two for GNB8) are marked with open black circles whereas the filled circles are the axes calculated as described above for all boundaries. It is seen that the measured as well as calculated axes lie quite close to the theoretical predictions. In particular the axes for GNB1 and 2 with dislocations coming only from the (111) plane lie close to the [111] axis predicted in Equation (13), which is the only possibility with two sets of dislocations. In each case the calculated misorientation axis is also in agreement with the theoretical axis expected based on the qualitative comparison of the experimental and predicted dislocation networks.

The misorientation angles have been calculated based on the average ratio of the lengths of the calculated A and B vectors. In all cases the angles were of the order of $0.5^{\circ}$, which agrees well with the values in the range $0.43-0.62^{\circ}$ measured for GNBs 7 and 8 . 


\section{Discussion}

\subsection{LEDS character}

According to the LEDS principle the dislocations assemble in structures to obtain the lowest energy configuration that is accessible to them. While this principle and the Frank equation has previously been applied in the analysis of dislocation boundaries, the present study is to the authors' knowledge the first to conduct a comprehensive prediction of all the possible configurations aligned with $\{111\}$ resulting from a specific slip geometry, in this case two sets of coplanar systems. The analysis is conducted by i) taking all of the available dislocations into account, ii) considering that at room temperature climb is not to be expected and iii) considering dislocation reactions in the boundary.

Two main categories of predictions were found, one consisting of dislocations from only one slip plane and the other containing dislocations from both active planes. This is in agreement with the experimental data from the previous paper. Apart from the trivial symmetry between the two active slip planes, two symmetrically equivalent variants of the second category containing dislocations from both slip planes were found. The qualitative comparison of the theoretically predicted solutions with experimental data in terms of the dislocation network morphology confirmed the existence of all of the theoretically predicted morphological network classes in Figure 2, Figure 3 and Figure 4 (right) including dislocation reactions to further lower the energy of the dislocations.

The quantitative analysis of whether the measured dislocation content fulfils the Frank equation when also taking into account the experimentally observed deviations from the exact slip plane also reveals good agreement, in the sense that the left and right sides of the Frank equation match each other well. The misorientation axes calculated from the experimental data are further in good agreement with the measured ones.

The measured data does not contain the sign of the dislocations and based on the measurements alone it can therefore not be determined whether the boundary contains redundant dislocations of the same Burgers vector but of opposite sign. The quantitative analysis is sign sensitive and in all cases the signs have been selected to best match the Frank equation. The fact that good agreement has been achieved shows that redundant dislocations of the same Burgers vector but opposite signs are not present in large densities in the GNBs.

The results clearly show that the dislocation boundaries are at least very close to being LEDS structures. This conclusion is in full agreement with a previous comparison of predicted and observed dislocation networks in the Type 2 cell structure of tensile deformed aluminium grains with the tensile axis near $\langle 100>[40]$.

\subsection{Boundary formation mechanism}

The analysis conducted was based on the assumption that the dislocations move by glide, i.e. do not move out of the slip planes. This raises the question of how the dislocations coming from the slip plane with which the GNB aligns become incorporated in the boundary. One possibility may be that the GNB is the result of a local slip concentration on the boundary plane and that the dislocations gliding in this plane interact to form the boundary network. As suggested by Jackson [41] dislocations gliding on 
physical slip planes close to the actual boundary plane may experience double cross slip to produce a dipolar wall aligned closely with the slip plane. This theory is consistent with the observation that the experimentally determined boundary planes always deviate a few degrees from the exact slip plane (the deviation for the presently analysed GNBs is $4-18^{\circ}$ ). A dipolar wall will, however, not be associated with a crystallographic misorientation and it will contain many redundant dislocations, even though additional dislocations gliding into the boundary may produce a misorientation.

Another possibility is that the small deviation of the GNB from the slip plane is necessary for the GNB to evolve as the deviation enables gliding dislocations to intersect with and accumulate in the GNB. As a consequence the dislocation lines must be the intersection line between the exact slip plane and the boundary plane. Finally, all dislocations may be transported to the boundary by cross slip. In this case the dislocation lines must be the intersection lines between the boundary plane and the cross slip plane.

In order to distinguish between the two last mechanisms, involving glide or cross slip, the experimental dislocation lines are compared with the intersection lines between the boundary plane and i) the slip and ii) cross slip planes. The comparison is conducted by means of the angle between the experimental dislocation line and the two intersection lines for b1 and $\mathbf{b 2}$ in Figure 11. Small uncertainties in the determination of the experimental boundary plane give rise to significantly more scatter in the calculated intersection with the (111) slip plane than for the cross slip plane because the boundary plane is very close to (111). Nevertheless, the deviations to the intersection with the slip plane (mostly more than $40^{\circ}$ ) in the left part of the figure for GNBs aligned with (111), are much larger than those for the cross slip plane (mostly within $20^{\circ}$ ). This situation is reversed in the right part of the figure for GNBs 5, 6 and 8 aligned with (11 $\overline{1})$, where the experimental dislocation lines match the intersection with the slip plane well $\left(<10^{\circ}\right.$ deviation). The corresponding analysis for dislocations of $\mathbf{b 4}$ and $\mathbf{b 5}$ with appropriate interchange of (111) and (11 $\overline{1})$ reveals the same and is not included here. Based on this analysis it is clear that dislocations gliding on the slip plane, which is not aligned with the GNB, glide directly into the boundary, as also assumed in the present derivations based on the Frank equation. The small deviations between the observed dislocation lines and the intersection line between GNB plane and the cross slip plane for the dislocations gliding on the planes with which the boundary aligns agrees well with the hypothesis that these enter by cross slip. It is noted that the Schmid factors for the systems on the cross slip planes have values of 0.2 to be compared with 0.4 for the slip planes, whereas all other Schmid factors have values of zero in the present crystallographic orientation. 


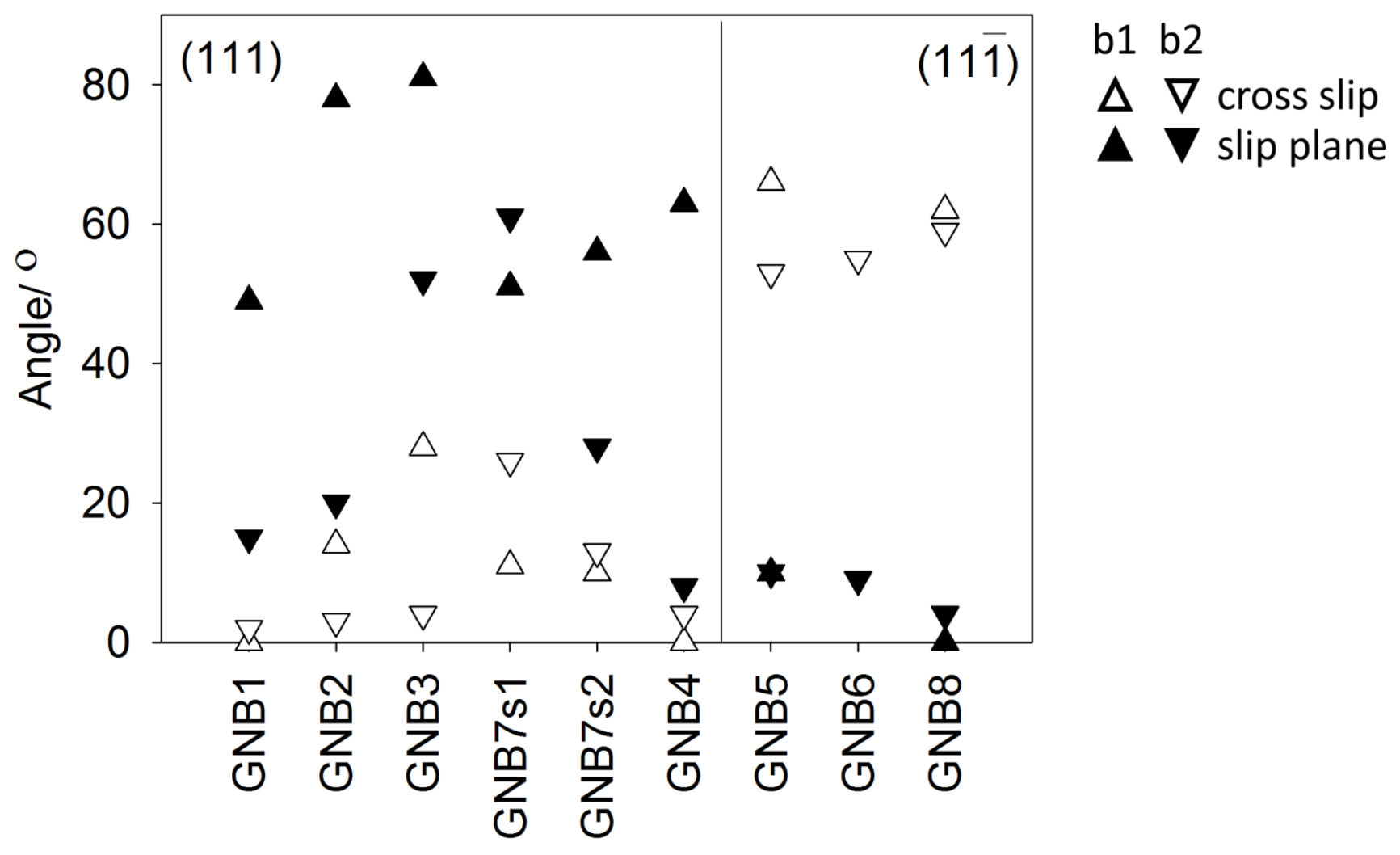

Figure 11. Angle of the experimental dislocation lines of b1 (upward triangles) and b2 (downward triangles) to the intersection lines of the boundary with the slip plane (filled symbols) and cross slip plane (open symbols).

Local interactions between the dislocations in the GNBs are responsible for the production of Lomer locks of $\mathbf{b 6}$ for boundaries constructed from more than two sets of dislocations. For boundaries constructed from two sets of dislocations a third dislocation of $\mathbf{b} \mathbf{3}$ is the reaction product. In this way the GNBs can contain all six Burgers vectors of the $1 / 2<110\rangle$ type even though only four of these participate in the slip system activity. This also explains the observation that slip systems $\mathbf{b 3}$ and $\mathbf{b 6}$ having Schmid factor values of zero are involved in the experimentally investigated $45^{\circ}$ ND rotated Cube orientation.

The role of cross slip for boundary formation in aluminium is also illustrated by comparison with the network of a single GNB segment observed in a Brass oriented grain in 7.5\% rolled copper [39]. The GNB alignment and misorientation as well as the Burgers vectors in that network are similar to the presently analysed observations in aluminium but none of the dislocations were screws. Variations in the visual sharpness of the boundaries in fcc metals have further been reported to scale with the propensity for cross slip $[42,43]$, whereas the grain orientation and slip system dependence of the GNB alignment in general is similar [14]. 


\section{Conclusions}

For the specific slip geometry of two sets of coplanar systems (i.e. a total of four systems) in aluminium, the range of dislocation networks in boundaries aligned with one of the two active slip planes has been predicted from the Frank equation for boundaries free of long-range elastic stresses. Detailed comparison with experimental data for eight dislocation boundaries in cold-rolled aluminium grains of the $45^{\circ} \mathrm{ND}$ rotated Cube orientation was conducted.

1. The boundaries are essentially Low Energy Dislocation Structures (LEDS):

- The close agreement with the Frank equation demonstrates that the boundaries are largely free of long-range elastic stresses.

- Dislocations in the boundary react to further lower their energy while still fulfilling the Frank equation.

2. Several types of networks were predicted:

- Networks with dislocations from two, three and four of the active slip systems are possible. Through dislocation reactions all six $1 / 2<110>$ Burgers vectors in fcc metals are represented.

- Linear combinations of the identified networks are also possible. The predicted range thus spans from pure tilt over mixed tilt/twist to pure twist boundaries.

3. Qualitatively and quantitatively, the experimental networks match the predictions:

- Qualitatively, the morphology of the experimental networks is similar to the predictions.

- Quantitatively, the experimentally determined dislocation content, in terms of Burgers vectors, dislocation lines and densities, as well as the misorientation axes are in close agreement with the Frank equation and match the predictions.

- All experimentally observed dislocations are needed, i.e. redundant dislocations are not present in significant densities.

4. Analysis of the experimental dislocation lines shows that:

- Dislocations gliding on the slip plane with which the boundary aligns cross slip into the boundary.

- Dislocations gliding on the other slip plane glide into the boundary directly. 


\section{Funding}

This work was supported by the Danish National Research Foundation (Grant No. DNRF86-5) and the National Natural Science Foundation of China (Grant No. 51261130091) for the DanishChinese Center for Nanometals, within which part of this work was performed; the Danish Independent Research Council | Technology and Production Sciences grant no. DFF - 133500220.

\section{References}

[1] N. Hansen, X. Huang, G. Winther. Mater. Sci. Eng. A 494 (2008) 61.

[2] D. Juul Jensen, N. Hansen. Acta Metall. Mater. 38 (1990) 1369.

[3] B. Peeters, M. Seefeldt, C. Teodosiu, S.R. Kalidindi, P. Van Houtte, E. Aernoudt. Acta Mater. 49 (2001) 1607.

[4] ZJ . Li, G. Winther, N. Hansen N. Acta Mater. 54 (2006) 401.

[5] G. Winther. Scr. Mater. 52 (2005) 995.

[6] B . Liu, D . Raabe, P. Eisenlohr, F . Roters, A . Arsenlis, G . Hommes. Acta Mater. 59 (2011) 7125.

[7] H . Mughrabi. Acta Metall. 31 (1983) 1367.

[8] B. Bay, N. Hansen, D . Kühlmann-Wilsdorf. Mater. Sci. Eng. A 113 (1989) 385.

[9] R. Madec, B . Devincre, L. Kubin. Philos. Mag. A 47 (2002) 689.

[10] B . Jakobsen, H.F. Poulsen, U . Lienert, J . Almer, S.D . Shastri, H.O . Sørensen, C . Gundlach, W. Pantleon. Science 312 (2006) 889.

[11] N . Hansen, X . Huang, W . Pantleon, G . Winther. Philos. Mag. 86 (2006) 3981.

[12] B . Bay, N. Hansen, D.A . Hughes, D . Kuhlmann-Wilsdorf. Acta Metall. Mater. 40 (1992) 205.

[13] G . Winther, X . Huang, N . Hansen. Acta Mater. 48 (2000) 2187.

[14] X. Huang, G. Winther. Philos. Mag. 87 (2007) 5189.

[15] Q . Liu, D . Juul Jensen, N . Hansen. Acta Mater. 46 (1998) 5819. 
[16] I . Gutierrez-Urrutia, D . Raabe. Acta Mater. 59 (2011) 6449.

[17] A.S . Taylor, P . Cizek, P.D . Hodgson. Acta Mater. 60 (2012) 1548.

[18] G.M . Le, A . Godfrey, C.S. Hong, X . Huang, G . Winther. Scr. Mater. 66 (2012) 359.

[19] B. Peeters, B. Bacroix, C. Teodosiu, P . Van Houtte, E . Aernoudt. Acta Mater. 49 (2001) 1621.

[20] A . Haldar, X . Huang, T . Leffers, N . Hansen, R.K . Ray. Acta Mater. 52 (2004) 5405.

[21] S . Wang, M.P. Wang, C . Chen, Z . Xiao, Y.L . Jia, Z . Li, Z.X . Wang. Mater. Charact. 91 (2014) 10.

[22] G. Winther, X . Huang. Philos. Mag. 87 (2007) 5215.

[23] G. Winther. Acta Mater. 56 (2008) 1919.

[24] F.X . Lin, A . Godfrey, G . Winther. Scr. Mater. 61 (2009) 237.

[25] Z.S . Basinski, S.J . Basinski. Philos. Mag. 9 (1964) 51.

[26] J.W . Steeds. Proc. R. Soc. A 292 (1966) 343.

[27] D.A . Hughes, S.M.A . Khan, A . Godfrey, H.M . Zbib. Mater. Sci. Eng. A 309 (2001) 310.

[28] G. Winther, D . Juul Jensen, N. Hansen. Acta Mater. 45 (1997) p. 5059.

[29] G. Winther. Acta Mater. 51 (2003) p. 417.

[30] Z.J. Li, A . Godfrey, Q. Liu. Acta Mater. 52 (2004) p. 149.

[31] C.S. Hong, X. Huang, G. Winther. Philos. Mag. 93 (2013) p. 3118.

[32] K. Morii, H. Mecking, Y. Nakayama. Acta Metall. 33 (1985) p. 379.

[33] A. Godfrey, D. Juul Jensen, N. Hansen. Acta Mater. 46 (1998) p. 823.

[34] H. Paul, J. Driver, C. Maurice, M. Miszczyk, D. Piot. Arch. Metall. Mater. 54 (2009) p. 65.

[35] G. Winther, X. Huang, A. Godfrey, N, Hansen. Acta Mater.52 (2004) p. 4437.

[36] F.C. Frank. The resultant content of dislocations in an arbitrary intercrystalline boundary. In: Symp. Plast. Deform. Cryst. Solids. Mellon Institute of Industrial Research, Pittsburgh1(950). p. 150. 
[37] J.P. Hirth, J. Lothe. Theory of Dislocations. McGraw-Hill, New York (1968).

[38] J.A. Wert, Q. Liu, N. Hansen. Acta Metall. 43 (1995) p. 4153.

[39] R.J. McCabe, A. Misra, T.E. Mitchell. Acta Mater. 52 (2004) p. 705.

[40] Y. Wei, A. Godfrey, W. Liu, Q. Liu, X. Huang, N. Hansen, G. Winther. Scr. Mater. 65 (2011) p. 355.

[41] P.J. Jackson. Prog. Mater. Sci. 29 (1985) p. 139.

[42] P. Landau, D. Mordehai, A. Venkert, G. Makov. Scr. Mater. 66 (2012) p. 135.

[43] P. Landau, G. Makov, R.Z. Shneck, A. Venkert. Acta Mater. 59 (2011) p. 5342. 